\title{
Polymer content and particle size effects on polymer-bonded Terfenol-D/PZT magnetoelectric composites
}

\author{
Yang Song a,b, ${ }^{\text {, }}$, Bo Liu ${ }^{\text {a, }}$, De'an Pan ${ }^{\text {a }}$, Lirong Xu ${ }^{\text {a }}$, Alex A. Volinsky ${ }^{\text {b }}$, Shengen Zhang ${ }^{\text {a }}$ \\ ${ }^{a}$ Institute for Advanced Materials and Technology, University of Science and Technology \\ Beijing, Beijing 100083, China \\ ${ }^{\mathrm{b}}$ Department of Mechanical Engineering, University of South Florida, Tampa, Florida 33620, \\ USA
}

\begin{abstract}
Polymer-bonded cylindrical Terfenol-D/PZT magnetoelectric (ME) composites have been prepared with varying polymer contents and Terfenol-D particle sizes. The axial ME voltage coefficient, $\alpha_{\mathrm{E}, \mathrm{A}}$, was investigated for studying the effects of polymer content and particle size on the composite ME performance. The Terfenol-D particles with four different size ranges $(<75 \mu \mathrm{m}, 75-100 \mu \mathrm{m}, 100-150 \mu \mathrm{m}$ and 150-180 $\mu \mathrm{m})$ were used. Additionally, the epoxy content of the composites was studied with five different weight ratios of $9 \mathrm{wt} \%, 12 \mathrm{wt} \%, 14 \mathrm{wt} \%, 16 \mathrm{wt} \%$ and $19 \mathrm{wt} \%$. The composite with an epoxy content of $0.14 \mathrm{wt} \%$ and $100-150 \mu \mathrm{m}$ particle range exhibited better overall ME performance. This investigation promotes selecting the appropriate polymer content and particle size to optimize fabrication of the polymer-bonded ME

\footnotetext{
* Corresponding authors.

E-mail addresses: yang@mail.usf.edu (Y. Song), liubo@ustb.edu.cn (L. Bo).
} 
composites for high ME performance to meet practical applications.

Keywords: Magnetoelectric composite; Terfenol-D; polymer content; particle size.

\section{Introduction}

The magnetoelectric (ME) effect is defined as the induced dielectric polarization under applied magnetic field $(H)$ through interfacial strain coupling of the two phases, or as the induced magnetization in the presence of an applied electric field [1]. Magnetoelectric composites made by combining piezoelectric (PE) and piezomagnetic (PM) materials can lead to remarkable ME effects at room temperature, compared with the single phase magnetoelectrics [2, 3]. Recently, ME composites have drawn much attention as a popular research topic because of the excellent ME performance at room temperature for potential applications in multifunctional devices, such as memory devices, tunable microwave devices, sensors [4-6].

Giant magnetostrictive material, Terfenol-D $\left(\mathrm{Tb}_{1-\mathrm{x}} \mathrm{Dy}_{\mathrm{x}} \mathrm{Fe}_{2-\mathrm{y}}\right)$, alloy is one of the best PM material candidates for ME composites [7]. However, some Terfenol-D properties hinder ME composite applications, including low mechanical strength, high eddy current losses at high working frequencies and fabrication size limits [8]. To solve the aforementioned problems, polymer-bonded ME composites are considered to have distinct advantages [9]. They are highly flexible, non-brittle and allow simple manufacturing processes at room temperature with various shapes and sizes. Polymer-bonded Terfenol-D ME composites that were earlier reported by Cewen Nan 
et al. Ma and Nan [10] demonstrated Terfenol-D-epoxy (TDE) medium as a PM phase for the ME composite by using 73 vol\% epoxy binding with Terfenol-D particles at room temperature to solve the brittleness and high eddy current losses problems of the Terfenol-D alloy. Zuo and Pan et al. [11] realized that the polymer phase content is directly influencing the ME properties as a non-functional phase. They fabricated Terfenol-D/PZT laminated composites with 97 wt\% Terfenol-D particle (randomly distributed sizes $<180 \mu \mathrm{m})$ and $3 \mathrm{wt} \%$ epoxy binder, using warm compaction $\left(130^{\circ} \mathrm{C}\right.$, $154 \mathrm{MPa})$, which resulted in the excellent voltage coefficient performance. Based on these studies, the authors investigated a one-step compression method of TDE/PZT cylindrical ME composites, which were prepared by using $<180 \mu \mathrm{m}$ Terfenol-D particles and PZT cylinder with $12 \mathrm{wt} \%$ epoxy binder [12]. However, few studies have focused on the effects of polymer content and particle size on polymer-bonded magnetoelectric composites so far. In an effort to optimize the polymer-bonded magnetoelectric composites, polymer-bonded TDE/PZT cylinder ME composites with various polymer content and particle size have been investigated. The axial mode voltage coefficient $\left(\alpha_{\mathrm{E}, \mathrm{A}}\right)$ of the samples was examined.

\section{Experiment}

The Terfenol-D particles were obtained by crushing bulk Terfenol-D single crystal alloy in argon atmosphere. The XRD pattern of the particles is shown in Fig. 1 (a). The proposed Terfenol-D/PZT cylindrical ME composites were made up of the 
aforementioned Terfenol-D particles and the PZT cylinder. The PZT cylinder was polarized along the radial direction. The Terfenol-D particles were compacted by uniaxial compression in the PZT cylinder, fabricated at $2 \mathrm{MPa}$ pressure. The Terfenol-D-epoxy needed 24 hours for curing. Detailed preparation method and schematic of the Terfenol-D/PZT cylindrical composite are shown elsewhere[12]. The ME performance of the Terfenol-D/PZT composites was obtained using the ME measurement system, where constant $\left(H_{\mathrm{DC}}\right)$ and alternating $(\delta H)$ magnetic fields were applied in the axial mode. The ME voltage coefficient was calculated as $a_{\mathrm{E}}=$ $\delta V /\left(t_{\mathrm{PE}} \cdot \delta H\right)$, where $t_{\mathrm{PE}}$ is the thickness of PZT and $\delta H$ is the amplitude of the AC magnetic field generated by the Helmholtz coils. The AC current flowing through the coil with the applied magnetic field amplitude of $\delta H=1.2$ Oe was equal to $1 \mathrm{~A}$. The axial mode ME voltage coefficient, $\alpha_{\mathrm{E}, \mathrm{A}}$, was measured when $H_{\mathrm{DC}}$ and $\delta H$ were applied along the axial direction of the cylinder.

\section{Results and discussion}

The microstructure of crushed Terfenol-D particles after sieving was examined by X-ray diffraction (XRD). The XRD pattern in Fig. 1 (a) of the particles is almost identical to the standard Terfenol-D XRD pattern, meaning that the crystal phase was not changed during the fabrication process [13]. The Terfenol-D particles with four different size ranges $(<75,75-100,100-150$ and $150-180 \mu \mathrm{m})$ were obtained using a set of two sieves with different meshes. Hence, the $<75 \mu \mathrm{m}$ range indicates the particles 
which passed through the sieve with an opening size of $75 \mu \mathrm{m}$. The $75-100 \mu \mathrm{m}$ range indicates that the particles passed through a $100 \mu \mathrm{m}$ opening sieve, but were blocked by the $75 \mu \mathrm{m}$ sieve. The particle size distribution is ideally uniform after sieving, noted by Fig. 1 (b), for the 150-180 $\mu \mathrm{m}$ particle size range.
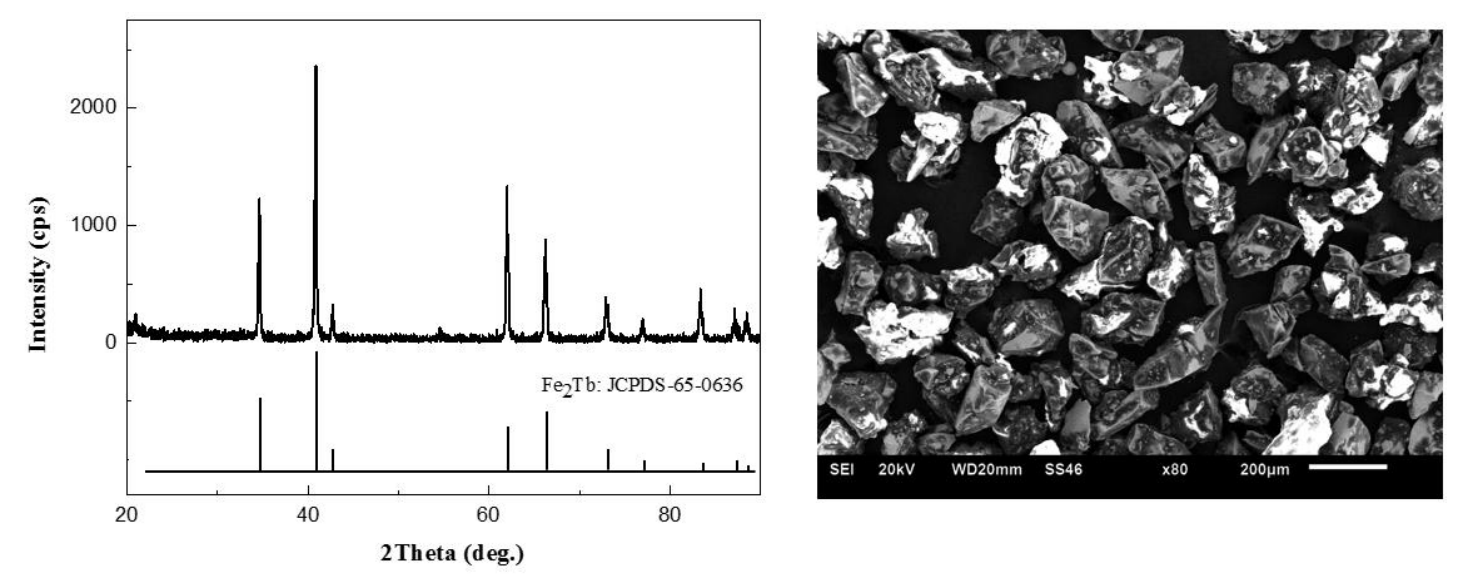

Fig. 1. (a) XRD pattern of the crushed Terfenol-D particles; (b) Terfenol-D particles with the $150-180 \mu \mathrm{m}$ size, observed by SEM.

The epoxy content of the composites was studied with five different weight ratios of $9 \mathrm{wt} \%, 12 \mathrm{wt} \%, 14 \mathrm{wt} \%, 16 \mathrm{wt} \%$ and $19 \mathrm{wt} \%$. Fig. 2 shows the ME voltage coefficient, $\alpha_{\mathrm{E}, \mathrm{A}}$, dependency on the volume ratio of various epoxy content with the 150-180 $\mu \mathrm{m}$ Terfenol-D particle size. As seen in Fig. 2, the ME voltage coefficient is greatly influenced by the epoxy content. The ME voltage coefficient reached the maximum value of $\alpha_{\mathrm{E}, \mathrm{A}}=3.37 \mathrm{~V} / \mathrm{cm} \cdot$ Oe when the epoxy content was $14 \mathrm{wt} \%$. The ME voltage coefficient was degraded when the epoxy content was below $14 \mathrm{wt} \%$. This could be due to the epoxy's inability to effectively play the binder and the lubricant roles at lower 
epoxy content [14]. When epoxy content increased over $14 \mathrm{wt} \%$, the ME performance decreased rapidly due to the dilution effect by the non-functional polymer phase [15].

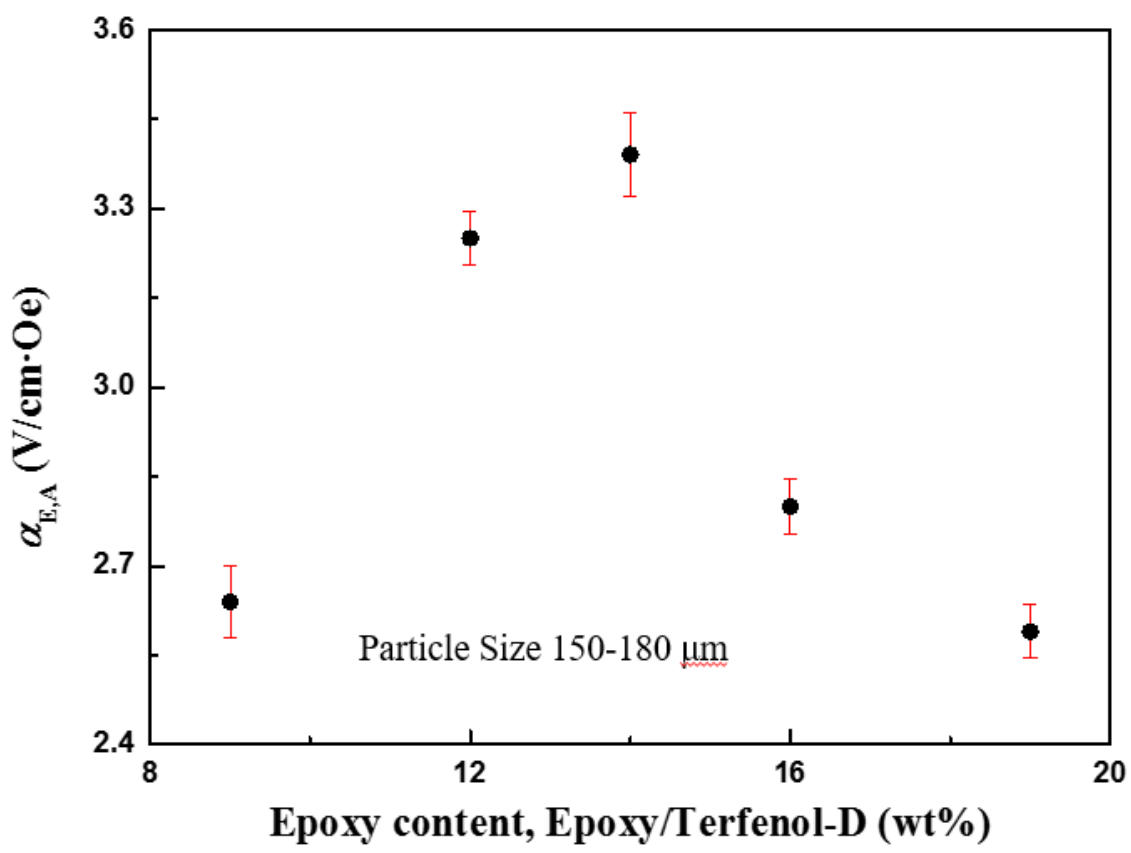

Fig. 2. The ME voltage coefficient, $\alpha_{\mathrm{E}, \mathrm{A}}$, dependence on the volume ratio of epoxy content. The results are for the sample with the particle size of $150-180 \mu \mathrm{m}$.

Fig. 3 shows the ME voltage coefficient, $\alpha_{\mathrm{E}, \mathrm{A}}$, particle size dependency for different Terfenol-D particle ranges of $<75 \mu \mathrm{m}, 75-100 \mu \mathrm{m}, 100-150 \mu \mathrm{m}$ and 150-180 $\mu \mathrm{m}$, with an epoxy content of 0.14 wt $\%$. As seen in Fig. 3, The ME voltage coefficient of the $<75 \mu \mathrm{m}$ particle size, $\alpha_{\mathrm{E}, \mathrm{A}}=2.1 \mathrm{~V} / \mathrm{cm} \cdot \mathrm{Oe}$, is much lower than others. When the particle size is between 100-150 $\mu \mathrm{m}$, the ME voltage coefficient is the highest, up to 3.4 $\mathrm{V} / \mathrm{cm} \cdot$ Oe. This result demonstrated that the ME voltage coefficient was also degraded when Terfenol-D particles were small. When particle size is between $100-180 \mu \mathrm{m}$, the 
composite can get roughly 1.6 times higher ME performance, compared with the particle size less than $75 \mu \mathrm{m}$.

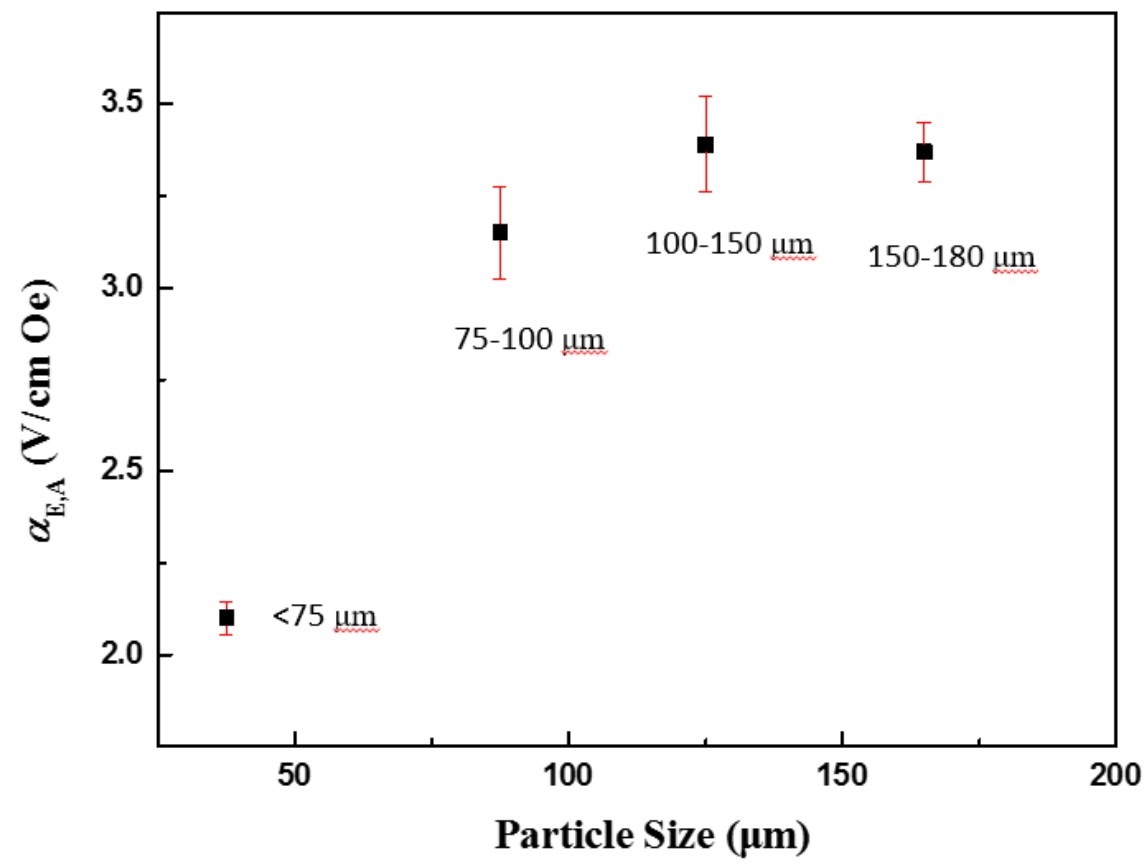

Fig. 3. The ME voltage coefficient, $\alpha_{\mathrm{E}, \mathrm{A}}$, dependence on the Terfenol-D particle size.

The results are for the samples fabricated with the epoxy content of $0.14 \mathrm{wt} \%$.

\section{Conclusions}

In summary, the axial ME voltage coefficient of the polymer-bonded cylindrical Terfenol-D/PZT ME composites was studied. The voltage coefficient can be distinctly influenced by both polymer content and particle size. The composite with an epoxy content of $0.14 \mathrm{wt} \%$ and $100-150 \mu \mathrm{m}$ particle sizes exhibited better overall $\mathrm{ME}$ performance. These findings promote selecting the appropriate polymer content and particle size to optimize fabrication of the polymer-bonded ME composites for high ME performance to meet practical applications. 


\section{ACKNOWLEDGEMENTS}

This work was supported by the National Natural Science Foundation of China (U1360202, 51472030 and 51502014), by the Beijing Nova program (Z141103001814006), by the Fundamental Research Funds for the Central Universities (Project No.: FRF-TP-14-001C1) and the China Postdoctoral Science Foundation Funded Project (Project No.: 2014M560885). Alex Volinsky acknowledges support from the National Science Foundation under the IRES 1358088 grant. Yang Song acknowledges support from the China Scholarship Council (CSC No.: 201506460047).

\section{References}

[1] Eerenstein W, Mathur ND, Scott JF. Nature 2006;442:759-65.

[2] Bibes M, Barthelemy A. Nat Mater 2008;7:425-6.

[3] Yang H, Zhang G, Lin Y, Wang F. Mater Lett 2015.

[4] Lee JH, Fang L, Vlahos E, Ke X, Jung YW, Kourkoutis LF, et al. Nature 2010;466:954-8.

[5] Shi Z, Tong Y, Deng S, Xue H, Yang S, Lu Y, et al. Appl Phys Lett 2013;103:032903.

[6] Liu B, Fang Y, Han Z, Yan S, Zhou W, Qian B, et al. Mater Lett 2016;164:425-7.

[7] Lu M-C, Mei L, Jeong D-Y, Xiang J, Xie H, Zhang Q. Appl Phys Lett 2015;106:112905.

[8] Lim S, Kim S, Kang S, Park J, Nam J, Son D. J Magn Magn Mater 1999;191:113-21.

[9] Martins P, Lanceros - Méndez S. Adv Funct Mater 2013;23:3371-85.

[10] Ma J, Shi Z, Nan CW. Adv Mater 2007;19:2571-3.

[11] Zuo ZJ, Pan D, Jia YM, Tian JJ, Zhang SG, Qiao LJ. J Alloy Compd 2014;587:287-9.

[12] Song Y, Pan Da, Wang J, Zuo Z, Zhang S, Liu B, et al. AIP Advances 2015;5:037104.

[13] Liu M, Li S, Zhou Z, Beguhn S, Lou J, Xu F, et al. J Appl Phys 2012;112:063917.

[14] Kadiyala AK, Bijwe J. Wear 2013;301:802-9.

[15] Liu J, Pan Z, Song X, Zhang Z, Ren W. J Appl Phys 2015;117:17A914. 
Polymer content and particle size effects were studied for ME composite

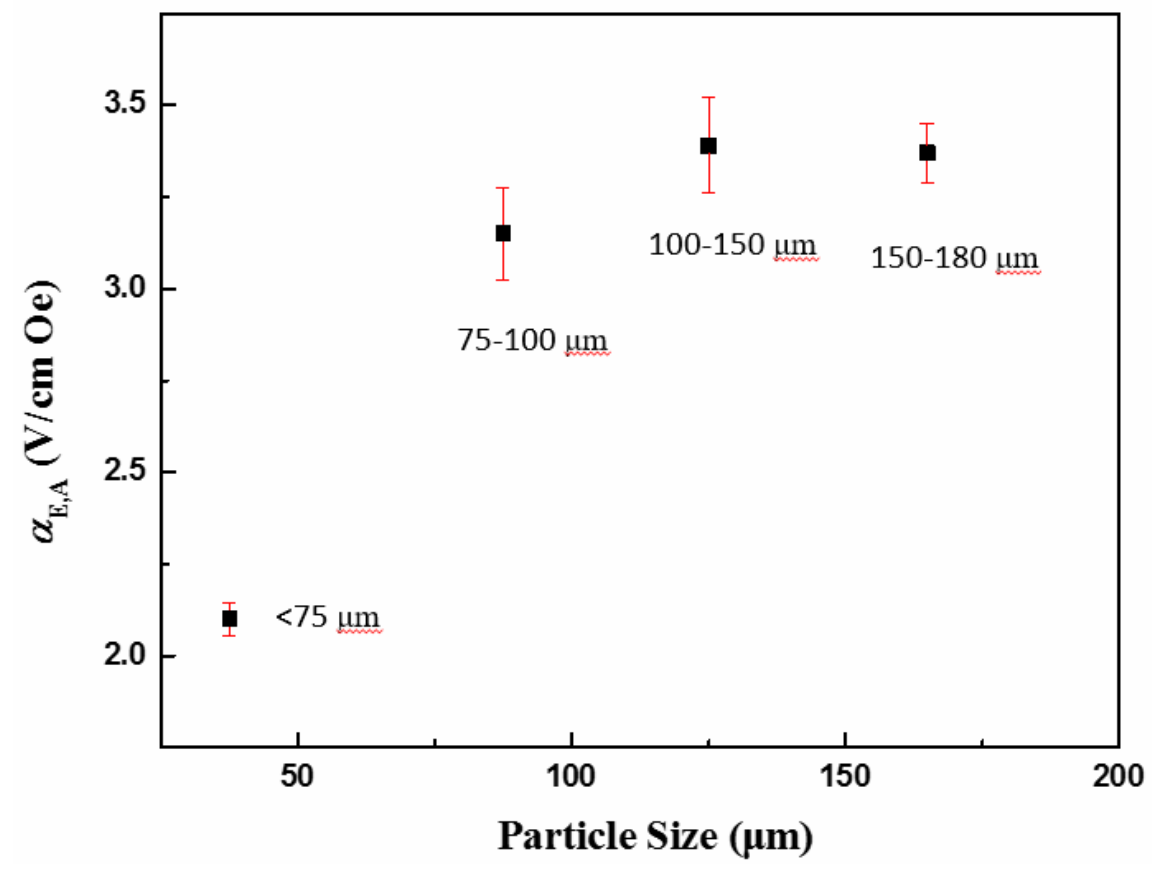

Best ME performance with 100-150 $\mu \mathrm{m}$ Terfenol-D size 